\title{
Archéopages
}

Archéopages

Archéologie et société

$44 \mid 2017$

Terrains vagues

\section{Le grand jet du parc à fontaines de Liancourt}

\author{
Jean-Louis Bernard
}

\section{OpenEdition}

Journals

Édition électronique

URL : https://journals.openedition.org/archeopages/1597

DOI : 10.4000/archeopages. 1597

ISSN : 2269-9872

\section{Éditeur}

INRAP - Institut national de recherches archéologiques préventives

\section{Édition imprimée}

Date de publication : 1 juin 2017

Pagination : 90-91

ISSN : 1622-8545

\section{Référence électronique}

Jean-Louis Bernard, «Le grand jet du parc à fontaines de Liancourt », Archéopages [En ligne], 44 | 2017 , mis en ligne le 01 juillet 2019, consulté le 03 juin 2021. URL : http://journals.openedition.org/ archeopages/1597; DOI : https://doi.org/10.4000/archeopages.1597 
Période

Époque moderne

\section{Le grand jet du parc à fontaines de Liancourt}

Jean-Louis Bernard

Inrap, Responsable d'opération

Site

Parc du château

Liancourt

Oise

Date

octobre 2016
1.Vestiges du bassin découvert en 2016 : la canalisation d'arrivée d'eau sous pression alimentait le bassin dont la surface et la forme sont indiquées par la margelle et le socle central, une autre canalisation permettant l'évacuation du trop-plein. 2504 : allée, 2505 : margelle, 2506: fond, 2507 : socle central, 2511: canalisation d'arrivée d'eau sous pression, 2604 : canalisation de trop-plein.

2. Restitution du plan du bassin, centré, à pans coupés longs de 6,80 m environ, et octogonal.
Encore très mal connu en 2011 lors des premiers diagnostics (Poussard, 2006 ; Bernard, 2011 ; Bourguignon, 2013), le parc à fontaines du château de Liancourt, qui fut l'un des plus beaux du royaume à partir du milieu du XVII ${ }^{\mathrm{e}}$ siècle, est désormais bien mieux compris. Il a été possible dans le cadre d'une collaboration entre l'Inrap, l'École nationale des arts et métiers et les Amis de l'Histoire, grâce aux fouilles successives et à l'étude très approfondie des sources archivées graphiques et textuelles (Bernard et al., 2013; Bernard, 2015; Bellagamba, 2015-2016), de proposer une restitution des quatre états successifs du jardin avant sa disparition à la fin du XIX ${ }^{\mathrm{e}}$ siècle et de son plan de distribution de l'eau. Le parc occupait toute la rive gauche de la rivière Brèche, affluente de l'Oise, jusqu'au sommet de la vallée où étaient captées les nombreuses sources fournissant une partie de l'eau aux nombreux jets, bouillons, canaux, bassins et fontaines (Mignot, 2016).

Un diagnostic mené en 2016 , en lien avec un projet de voirie départementale, a permis d'intéressantes observations complémentaires, notamment la découverte d'un bassin fort mal situé par les textes [ill. 1]. Il n'a malheureusement été observé que le dernier jour de l'opération et le temps a manqué pour le dégager aussi finement qu'il le méritait. La margelle n'est que partiellement conservée et sur une seule assise d'épaisseur. On observe une section droite large de $80 \mathrm{~cm}$ en moellons calcaires liés au mortier, visible sur une longueur de 4,60 m. On trouve par ailleurs l'empreinte dans le sédiment encaissant des parties disparues, ce qui permet de restituer le plan du bassin [ill. 2]. La margelle est contournée par un épandage de gravier qui forme une allée confortable large d'environ 2,70 m. Ce sédiment à base d'éclats de silex est bien reconnaissable, car présent sur l'ensemble du site où il a été systématiquement utilisé pour le traitement des niveaux de circulation. Le centre de l'aménagement est occupé par un massif maçonné constitué d'une épaisse masse de moellons noyés dans un mortier de chaux entourant un solide socle central fait de quatre très gros monolithes calcaires taillés dont subsistent l'assise inférieure et un bloc de l'assise supérieure. Le fond est constitué d'un épandage à peu près plan de sédiment naturel sableux, marqué par des dépôts tartreux avec quelques graviers et des déchets de mortier hydraulique. Il n'y a aucune étanchéité, fait étonnant qui avait déjà été remarqué au bassin des 7 fontaines (Bernard, 2015) : à l'évidence, ce secteur de fond de vallée est tant gorgé d'eau qu'il est inutile d'étanchéifier les bassins ! Deux tuyaux, composés de tubes en céramique, emboîtés et soutenus par un muret maçonné en moellons, étanchéifiés par une gangue de mortier, semblent liés à la structure.
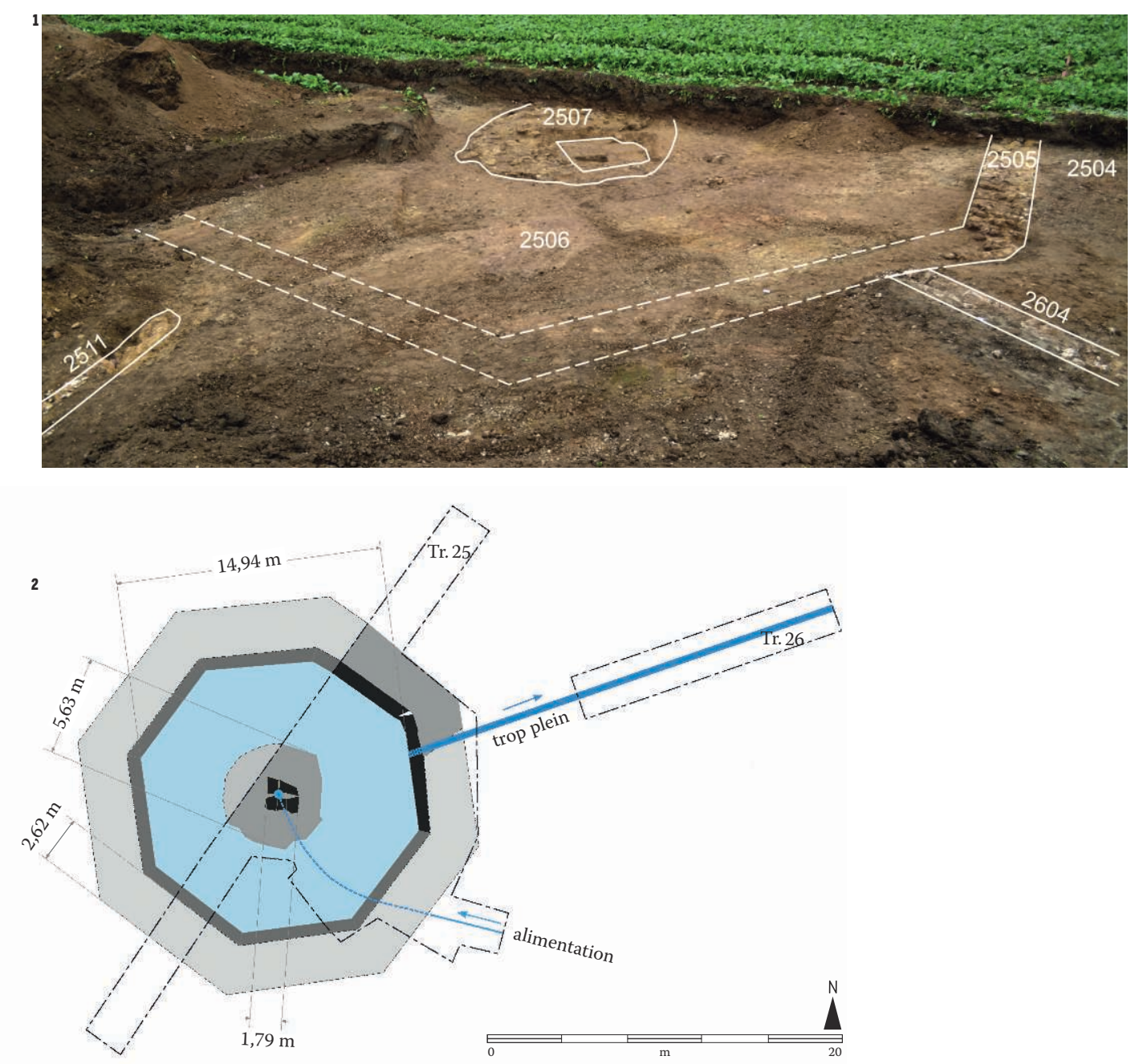


\section{Partie la mieux conservée du tuyau de la seconde moitié du xviI' siècle évacuant l'eau du bassin (2604 ; Diamètre intérieur : $18 \mathrm{~cm}$ ) vers le grand canal (situé sous la haie d'arbres derrière les véhicules). Il a été observê jusqu'au bord de la margelle sur une distance de $59,76 \mathrm{~m}$. \\ 5. L'emprise du diagnostic et le plan par masses de cultures, fin du xVIII' siècle, alors que le parc est abandonné. Le cercle non renseigné visible sous le bassin marque probablement le souvenir de ce dernier. Le grand canal est coloré en bleu.}

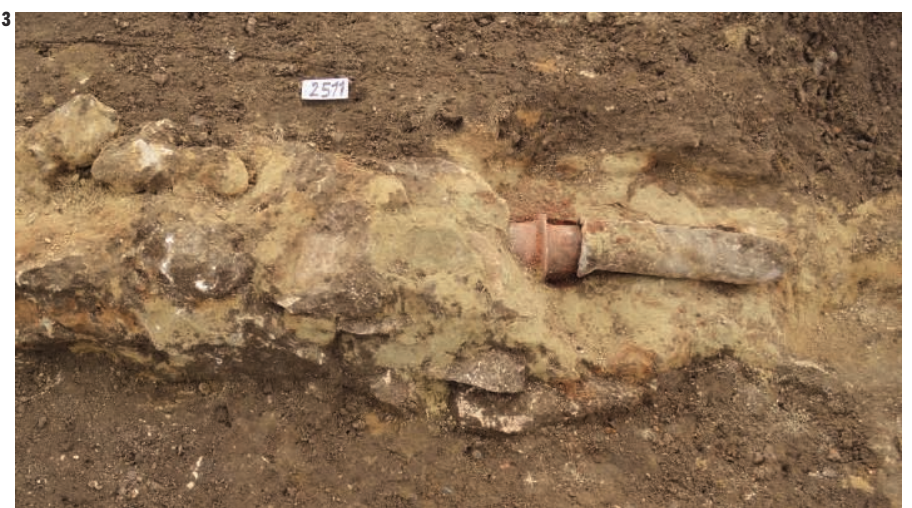

L'adduction est assurée par un tuyau qu'on retrouve sous l'allée extérieure. Il est prolongé par une courte section de tuyau en plomb, vestige du tube qui assurait la liaison avec la structure centrale [ill. 3]. Un gros tuyau sert d'exutoire [ill. 4].

La présence de ce qui ressemble beaucoup à un bassin avec fontaine centrale n'était pas attendue à cet endroit, à l'extrémité du parc, contre l'allée du fond. Le bassin le plus proche - «les Caillerins», représenté sur le plan Desmarest de 1743 n'est mentionné par aucune source textuelle connue. Cette identification est peu probable car les recollements de plans montrent un écart de $70 \mathrm{~m}$, ce qui paraît excessif même en tenant compte des incertitudes d'échelles. Par ailleurs, le plan d'archives figure clairement un bassin circulaire alors que nous découvrons un bassin octogonal. Une autre hypothèse envisagée est l'attribution du bassin découvert à la grande vasque du $\mathrm{XVI}^{\mathrm{e}}$ siècle en marbre de Carrare achetée au XVIII ${ }^{\mathrm{e}}$ siècle à Florence par le duc de la Rochefoucault et installée dans le parc. Son poids considérable et ses dimensions pourraient s'accorder avec le très fort massif de fondation découvert au centre du bassin. L'emplacement de cette vasque, conservée aujourd'hui dans le parc de la Rochefoucault en Charente, est incertaine car les descriptions de voyageurs sont imprécises. Jusquà présent, on est plutôt tenté de la positionner du côté du bassin des 7 fontaines.

L'hypothèse la plus conforme aux descriptions dont nous disposons consiste à rapprocher cette fontaine du grand jet du XVIII ${ }^{e}$ siècle. Les guides de voyage (Villiers, 1802, p. 509) suggèrent en effet que le grand jet du XVII ${ }^{e}$ siècle, qui était dans l'axe de la façade du château, a été déplacé au siècle suivant et dans le cadre du réaménagement du parc, en même temps que sa puissance de jaillissement était réduite à $15 \mathrm{~m}$. Loin d'être resté l'attraction majeure du parc, le grand jet serait donc repoussé à l'extrémité du jardin, au bord de l'allée périphérique [ill. 5]. Les canalisations ne figurent pas sur les plans, mais on peut supposer raisonnablement que la petite conduite forcée qui l'alimente vient de la machine dont la colonne d'eau est de l'ordre de $16 \mathrm{~m}$, et que le gros tuyau de tropplein rejetait son eau dans le grand canal distant de $140 \mathrm{~m}$. réinstallé dans un bassin octogonal,
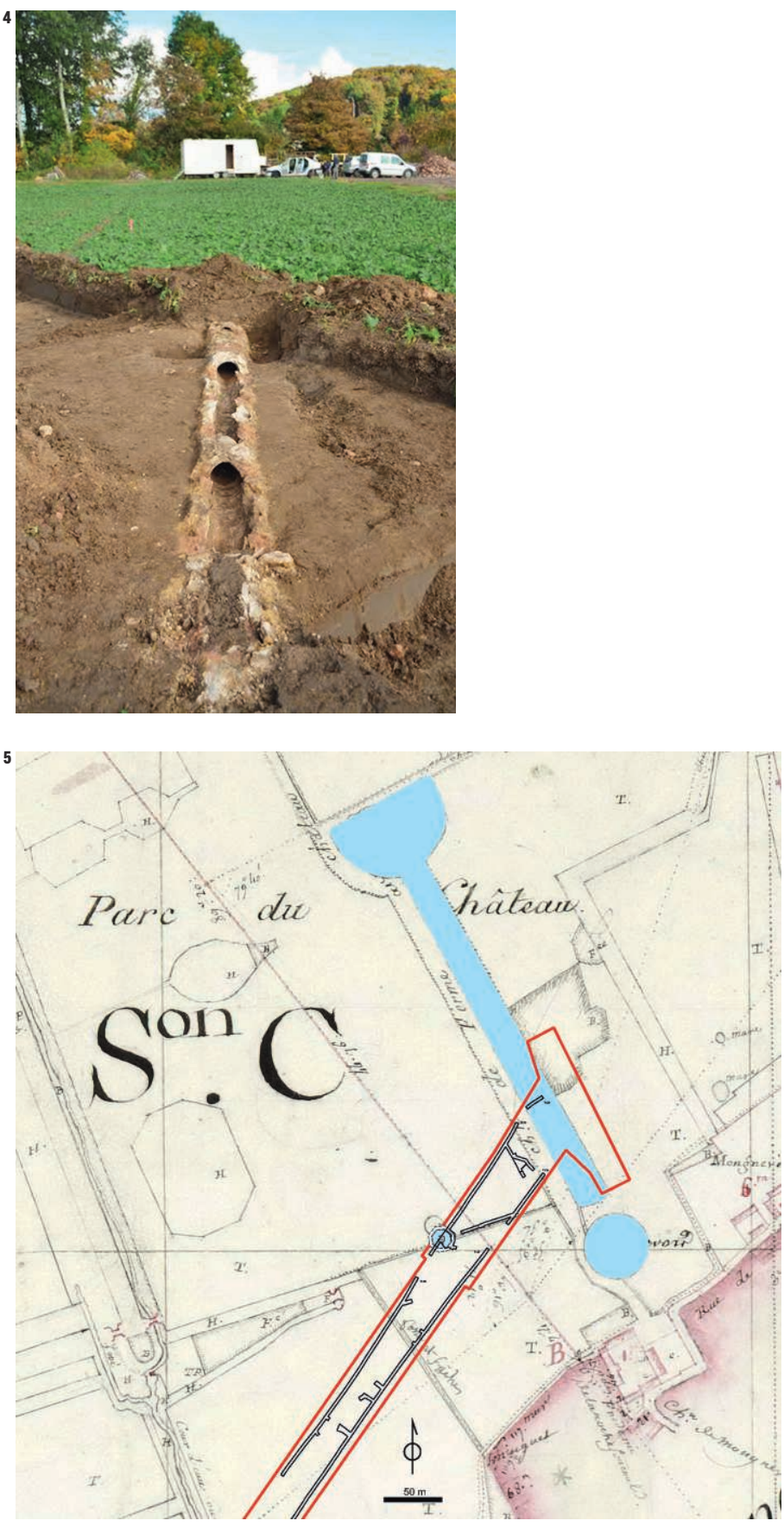

\section{Références bibliographiques}

VILLIERS P. 1802, Manuel du voyageur aux environs de Paris..., Paris, Favre, 2 vol.

Bellagamba F., 2015-2016, Plan général du château et du parc de Liancourt, Fondation des Arts et Métiers. http: //www.fondam.org/uploads/Fichiers/ jardinetchateauliancourt.pdf

BERNARD J.-L., 2011, Liancourt: avenue de l'île-de-France, rapport d'opération, Inrap-SRA Picardie, $77 \mathrm{p}$.

BERNARD J.-L., 2015, Liancourt le Parc, avenue Pierre Bérégovoy rapport d'opération, Inrap-SRA Picardie, $150 \mathrm{p}$
Bernard J.-L., Boulen M., Zeinoun P., DUMESNIL W., 2013, Liancourt, Oise, avenue de l'Île-de-France, rapport d'opération, Inrap-SRA Picardie, $290 \mathrm{p}$.

BourguignON A, 2013, "D'un clocher à l'autre, une histoire du château de Liancourt », Les Amis de l'Histoire, $\mathrm{n}^{\circ} 27$, nouvelle édition, $61 \mathrm{p}$.

Mignot M., 2016, « Quand Liancourt cultivait son jardin ", Arts et Métiers Mag, $\mathrm{n}^{\circ} 379$, p. 56-57.

POUSSARD R., 2006, " Les châteaux de Liancourt », Bulletin du Groupe d'Étude des Monuments et Euvres d'Art du Beauvaisis, n ${ }^{\circ}$ 129-130, p. 2-62. 